\title{
Starburst Galaxies as possible sources of UHECRs and neutrinos
}

\author{
Antonio Condorelli, ${ }^{a, b, *}$ Denise Boncioli, ${ }^{c, b}$ Enrico Peretti $^{d}$ and Sergio Petrera ${ }^{a, b}$ \\ ${ }^{a}$ Gran Sasso Science Institute, Via F. Crispi 7, 67100, L'Aquila, Italy \\ ${ }^{b}$ INFN/Laboratori Nazionali del Gran Sasso, via G. Acitelli 22, 67100, Assergi (AQ), Italy \\ ${ }^{c}$ Universita 'dell'Aquila, Dipartimento di Scienze Fisiche e Chimiche, Via Vetoio, 67100, L'Aquila, Italy \\ ${ }^{d}$ Niels Bohr International Academy, Niels Bohr Institute, University of Copenhagen, Blegdamsvej 17, \\ DK-2100 Copenhagen, Denmark \\ E-mail: antonio.condorelli@gssi.it, denise.boncioli@aquila.infn.it, \\ peretti@nbi.ku.dk, sergio.petrera@gssi.it
}

The coexistence of powerful accelerators of cosmic rays with intense background radiation fields creates unique conditions, where the Ultra-High-Energy Cosmic Rays (UHECRs) interactions could take place copiously and produce several secondary particles. In particular, such phenomena could explain the features observed in the UHECR spectrum measurements.

This scenario is explored extending SimProp, a simulation code for UHECR extra-galactic propagation, allowing us to compute the interactions inside and outside the source.

Inspired by recent results from the Pierre Auger Observatory on the anisotropy in the arrival directions of the highest energy cosmic-rays, the simulations are performed in the context of Starburst Galaxies, one of the most promising candidate sources. We model the interactive and diffusive processes in the region surrounding the Starburst Nucleus, taking into account interactions with background photons and the interstellar medium. The escaping cosmic-ray flux is then propagated to the Earth and compared with the experimental spectrum and composition measured by the Pierre Auger Observatory. The proposed method could be used to connect the features of the UHECR spectrum and composition at Earth to the parameters describing Starburst Galaxies. In addition, neutrinos directly produced in these sources can be compared to the measured IceCube flux, thus providing an additional way to constrain some source parameters.

$37^{\text {th }}$ International Cosmic Ray Conference (ICRC 2021)

July 12th - 23rd, 2021

Online - Berlin, Germany

\footnotetext{
${ }^{*}$ Presenter
} 


\section{Introduction: Source propagation model}

In the measured Ultra-High-Energy Cosmic Rays (UHECRs) energy spectrum, some features can be recognized: a hardening of the spectrum at the energy of $\simeq 5 \mathrm{EeV}$, called "ankle", and a suppression of the flux at $\simeq 40 \mathrm{EeV}$. Since the ankle was discovered, many interpretations have been addressed: one possibility allows the interpretation of this feature as the interplay of two extragalactic components, one more energetic, composed by intermediate to heavy nuclear species, with a hard spectral index, while the second one less energetic, lighter and with a softer spectral index [1]. A combined fit analysis, based on a two component scenario, is presented at this Conference by the Auger Collaboration [2].

On the other hand, in several works [3-6] it was shown how the post-processing of UHECRs via photo-disintegration of $\mathrm{CR}$ nuclei in the environment surrounding the source can naturally explain the entire spectrum and composition. In these models, the photo-disintegration process generally acts as a high-pass filter, permitting the highest energy cosmic-ray nuclei to escape unscattered while the lowest energy ones are disintegrated inside the source region, generating a pile-up of nucleons with energy $1 / A$ of the original nucleus of mass $A$. The particles escaping the source environment are then propagated through the intergalactic medium and then the diffuse fluxes are compared to the experimental data at Earth. For some choices of the parameters describing the source environment, these models, hereinafter called "source-propagation models", are able to describe the UHECR energy spectrum and mass composition at Earth [7] over a large energy raege. In this work we build a source-propagation model in a potentially interesting class of sources: Starburst Galaxies.

\section{Starburst Galaxies}

Starburst Galaxies (SBGs) are unique objects in the Universe characterized by a high rate of supernova explosions and star formation rate which can often be as high as $\sim 10^{2} \mathrm{M}_{\odot} \mathrm{yr}^{-1}$ [8]. As reported in [9], the higher the rate of star formation, the greater the infrared luminosity. Such a high supernova rate makes SBGs very promising factories for cosmic rays.

In several SBGs, the star forming region is observed to be localized in the central part of the galaxy with typical radial extension of $R \sim 0.2-0.5 \mathrm{kpc}$. The starburst environment also exhibits additional extreme conditions such as particle density higher than $n_{\mathrm{ISM}} \sim 10^{2} \mathrm{~cm}^{-3}[10]$ and infrared energy density $\left(U_{\mathrm{RAD}}\right)$ often higher than $10^{3} \mathrm{eV} \mathrm{cm}^{-3}$. The superposition of several supernovae and the intense star forming activity favor the existence of a powerful wind with estimated velocity $\left(v_{\mathrm{W}}\right)$ of about $\sim 10^{2} \div 10^{3} \mathrm{~km} \mathrm{~s}^{-1}$. The high level of turbulence in such a crowded environment suggests that SBGs can accelerate cosmic rays up to energies above hundreds of PeV [11-13]. In addition, the Pierre Auger Collaboration has found a correlation of UHECRs above $39 \mathrm{EeV}$ with a catalogue of 17 SBGs [14].

For simplicity, we choose to test the source-propagation model assuming that all the sources have the same parameters of a reference SBG, M82 [15]. Therefore M82 is used in this work as a starting prototype for our model; plausible ranges of values for the source parameters is considered, in order to find the best agreement to the experimental data. 

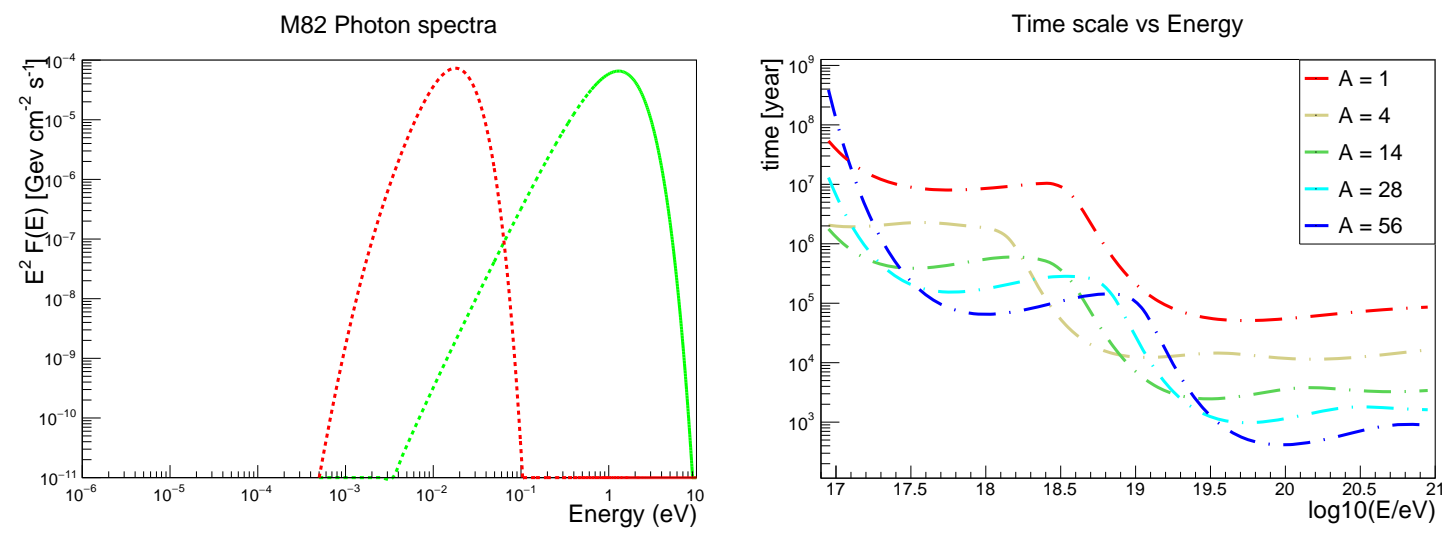

Figure 1: Left: Photon spectrum of M82, as inspired by [15]: thermal dust modified black bodies (red line) and optical star black body (green line). Right: Photo-interaction times in M82 for five different injected CR nuclear species.

\subsection{Photo-hadronic interactions in Starburst Galaxies}

As other different SBGs, the emission of M82 spans over several orders of magnitude in energy, as can be seen in figure 1 (left). The two most prominent photon fields in typical SBGs are black body for star light (green line in figure 1 (left)) and diluted black body in infrared (IR) for ultraviolet (UV) reprocessed by the dust (red line in figure 1 (left)), peaked respectively in the visible $\varepsilon_{\mathrm{opt}}=0.33 \mathrm{eV}$ and in the infrared range $\varepsilon_{\mathrm{IR}}=3 \cdot 10^{-3} \mathrm{eV}$. In the simulation code used, named SimProp [16], assuming a certain photon field $n_{\gamma}$, the inverse of the interaction time can be written such as [17] :

$$
\frac{1}{\tau_{\mathrm{int}}}=\frac{1}{2 \Gamma^{2}} \int_{\epsilon_{\min }^{\prime}}^{2 \Gamma_{\epsilon}} \int_{\epsilon^{\prime}=0}^{+\infty} \frac{n_{\gamma}(\epsilon)}{\epsilon^{2}} d \epsilon \sigma\left(\epsilon^{\prime}\right) \epsilon^{\prime} d \epsilon^{\prime}
$$

where $\sigma\left(\epsilon^{\prime}\right)$ is the differential cross section for a specific process and $\Gamma$ is the Lorentz factor of the interacting nucleus. Note that primed symbols (e.g. $\epsilon^{\prime}$ ) refer to quantities in the nucleus rest frame, while unmarked symbols refer to quantities in the laboratory frame. The interaction times from equation 1 are plotted in figure 1 (right); looking at heavy nuclei, the dip at low energies corresponds to the photo-disintegration on the visible light, while at higher energies the interaction time is dominated by the infrared spectrum. The original version of the SimProp code was adapted in order to include interactions inside the source environment.

\subsection{Hadronic interactions in Starburst Galaxies}

Spallation processes are negligible for the extra-galactic propagation, due to the low density of the gas; on the contrary, for UHECRs in SBGs, interactions with matter have to be taken into account. To this aim, we include in SimProp a parametric model based on Sibyll 2.3d [18], which is an event generator designed for Monte Carlo simulations of atmospheric cascades at ultra-high energies. Starting from Sibyll2.3d outcomes, it is possible to parametrize the relevant interaction parameters as a function of the energy $E$ and mass $A$ of the interacting particle. The key quantities are the inelastic spallation cross sections $\sigma_{p p}$ and $\sigma_{p A}$, the fragmentation of nuclei after their 

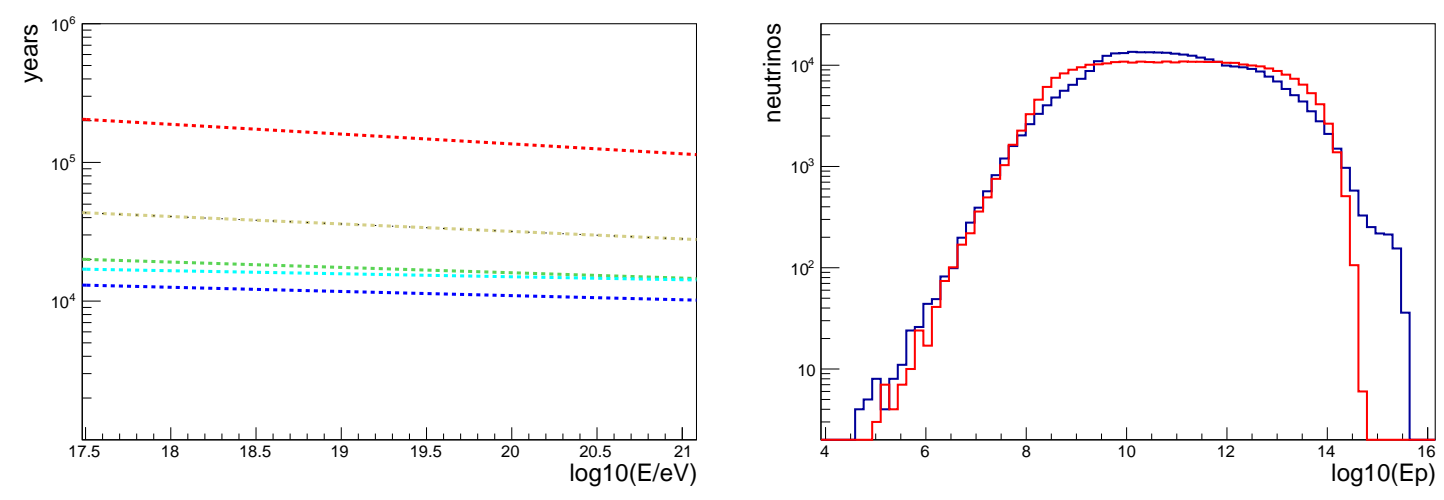

Figure 2: Left: Spallation times for different nuclear species (the legenda is the same of figure 1). Right: Neutrinos energy distributions for Sibyll2.3d (blue) and the parametric model (red) used in this work $\left(E=10^{17} \mathrm{eV}, A=28\right)$.

interaction and the multiplicity of secondary particles. Using the cross section outcomes it is possible to compute the spallation interaction times, shown in figure 2 (left).

A comparison between Sibyll2.3d and our parametric model has been performed, in order to evaluate the agreement of emitted secondary particles. Fixing a certain energy $\left(E=10^{17} \mathrm{eV}\right)$ and mass $(A=28)$ of the interacting particle, the comparison is performed in figure 2 (right), where the neutrino fluxes are shown. It is possible to conclude that the two distributions are qualitatively consistent with each other and, for this reason, the parametric model is used for this analysis. Nonetheless, it is important to stress the simplifications which have been assumed: in the parametric model all the produced secondary particles are supposed to be pions. In addition, using Sibyl12.3d, it was found that the fragments distribution is more complex than the simplistic assumption of the parametric model; the approximation used therein (a single heavy fragment $A_{\text {frag }}$ in the final state and $A-A_{\text {frag }}$ free nucleons) influences our results is still under investigation. A final goal of this work consists of a code with Sibyll2.3d fully embedded in SimProp.

\subsection{Escape}

High energy particles populating an environment can be confined in it for a limited time before escaping. Indeed particles can leave the system as the result of advection in a wind or via diffusion. In particular, the advection timescale can be written as $t_{\mathrm{adv}}=R / v_{\mathrm{W}}$, where $R$ is the source size and $v_{\mathrm{W}}$ is the wind speed. The diffusion timescale reads: $t_{\mathrm{D}}=R^{2} / D$, where $D$ is the CR diffusion coefficient computed in the context of quasi-linear theory assuming Kolmogorov turbulence and a coherence length $l_{c} \sim 1 \mathrm{pc}$ for the magnetic field. The expression of the diffusion coefficient is $D=r_{L}^{1 / 3} l_{c}^{2 / 3} / 3$, where $r_{L}$ is the particle Larmor radius. In agreement with [19], we additionally consider the transition in the diffusion regime taking place when $r_{L} \gtrsim l_{c}$. In this energy range the diffusion coefficient is estimated as $D=D_{0}\left(r_{L} / l_{c}\right)^{2}$, where $D_{0}$ is the value of the diffusion coefficient computed at the energy $E_{0}$ such that $r_{L}\left(E_{0}\right)=l_{c}$.

We finally estimate the escape timescale as the minimum between the advection and the diffusion time, namely $t_{\mathrm{esc}}=\min \left[t_{\mathrm{adv}}, t_{\mathrm{D}}\right]$. In figure 3 we show the escape timescales compared to 


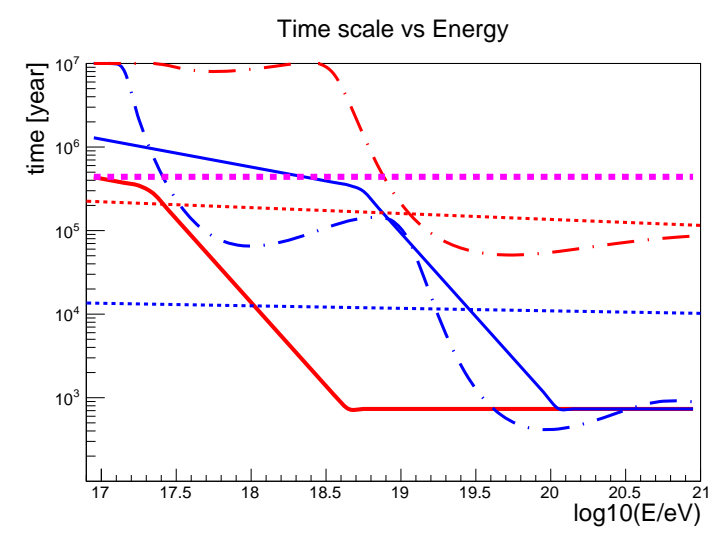

Figure 3: Timescales for M82 case: photo-hadronic interaction times (dashed-dot lines), spallation times (dashed lines) and diffusion times (solid lines) for protons (red) and iron nuclei (blue). The pink dashed line is the advection time.

the other typical timescales characterizing the system.

\section{Results}

The calculation of the interaction and escape probability have to be performed: if the probability of escape is dominant, the particle is not propagated anymore in the cascade; otherwise, the particle loses energy and mass according to the dominant process. Finally, the escaping flux is propagated through the extra-galactic medium and compared to the experimental data at Earth.

At this stage the source parameters, namely the radius of Starburst Nucleus (SBN), the IR luminosity and the ISM density, are fixed to the M82 ones. We then assume all the sources to be identical to M82, injecting a single nuclear species (silicon nuclei): a diffuse flux from these sources distributed as the star formation rate is propagated to the Earth and is compared to the measurements of the Pierre Auger Observatory, such as the energy spectrum and the mass composition observables as reported in [7]. It turns out that particles accelerated in the M82 environment do not provide a good agreement with the experimental data at Earth. At this point, moving from the M82 hypothesis, it is possible to search for a starburst prototype capable to describe the Auger data by scanning parameters at the source within plausible ranges. It was chosen to move the ISM density together with the IR luminosity, following the Kennicutt-Schimdt law [10], an empirical relation between the gas density and star formation rate. In addition, parameters related to the accelerated spectrum of CR are also varied. The best agreement found is shown in figure 4 and it corresponds to a prototype with a more intense IR photon field $\left(L_{\mathrm{IR}}=3.4 \cdot 10^{45} \mathrm{erg} / \mathrm{s}\right)$ with respect to M82, for what concerns source parameters, while for the spectral parameters at the acceleration hard spectra are preferred.

We additionally compute the expected neutrino flux for a certain choice of the source parameters. These are shown in figure 5: on the left for the M82 case, on the right for the configuration that best describes the UHECR data. In this analysis it is possible to distinguish neutrinos coming from interactions with the CMB and EBL (grey line) to the neutrinos produced in interactions with the 

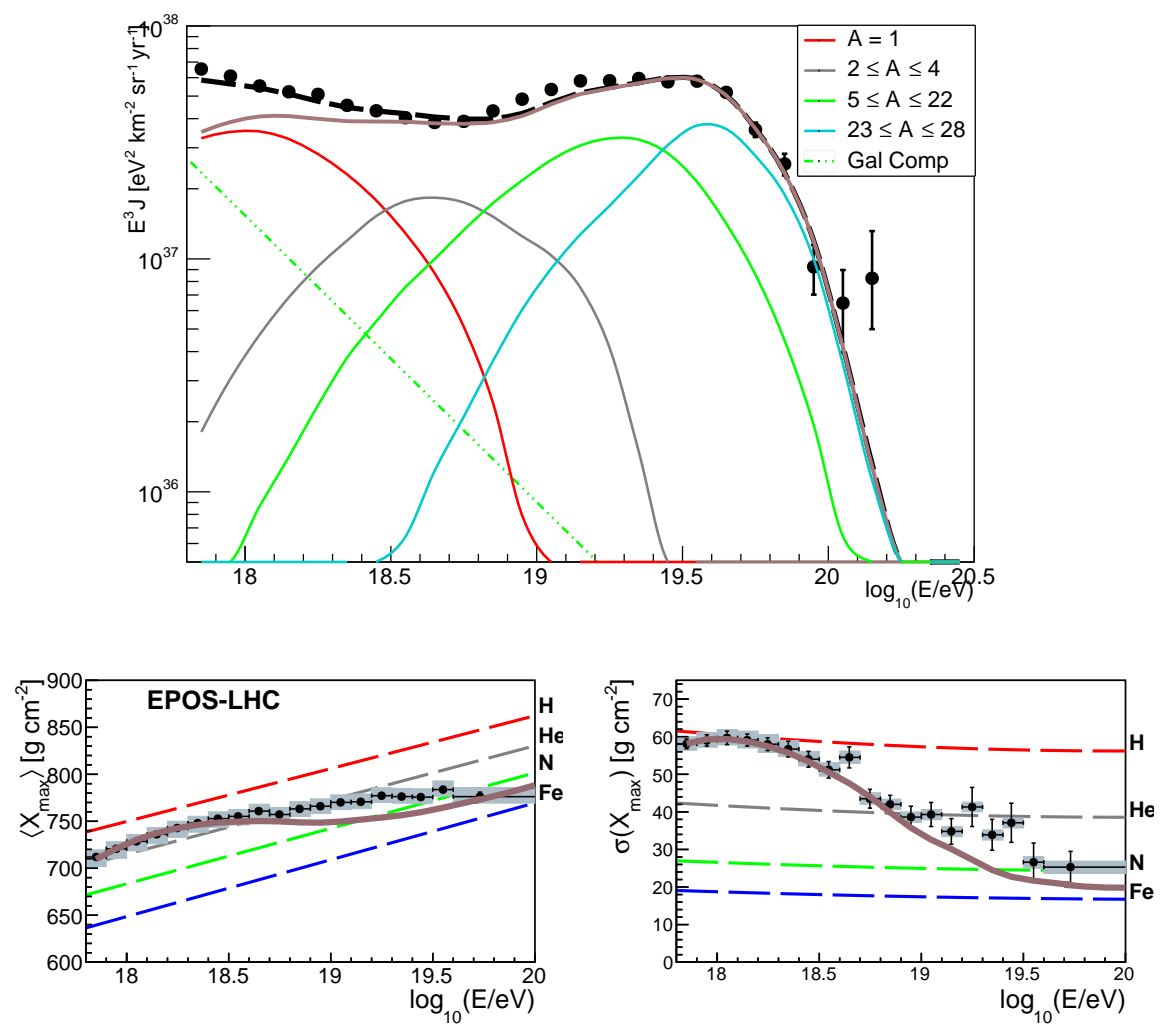

Figure 4: Expected UHECR spectrum (upper plot) and composition (lower plot) at Earth using the best configuration in the source parameter space, compared to measurements from the Pierre Auger Collaboration [7]. In the energy spectrum (upper plot), the dashed green line refers to the used galactic component, while the black dashed line refers to the total flux and the full colored lines to the fluxes grouped by mass.
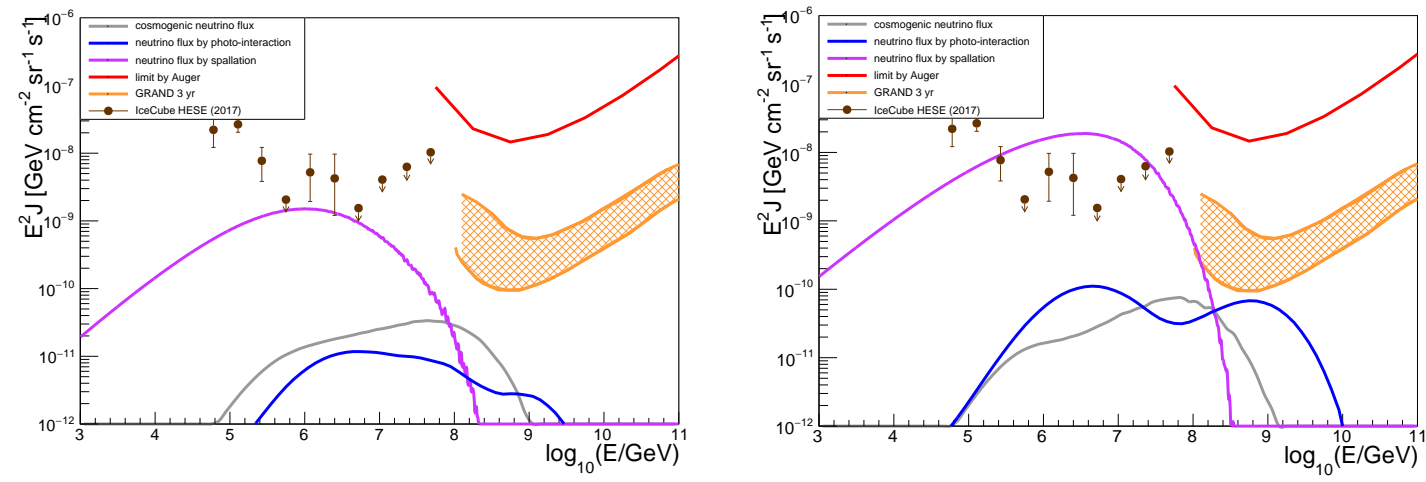

Figure 5: Neutrino fluxes for M82 case (left) and best case (right), compared to the IceCube neutrino flux [20], the GRAND expected limits after three years of operation [21] and to the limits for the cosmogenic neutrinos by the Pierre Auger Collaboration [22]. 
photons (blue line) surrounding the accelerator at the source and the neutrinos produced in hadronic interactions (magenta line), to be compared to the current astrophysical neutrino flux as measured by IceCube [20] and to the limits for the cosmogenic neutrinos [22]. Comparing the two plots, one can notice how the source-neutrino fluxes increase with increasing IR luminosity and ISM density. On the other side, the cosmogenic neutrinos are almost not affected by the source characteristics, as one could expect, and due to the UHECR mass composition and spectral parameters requested to reproduce the UHECR data, are way below the current limits for the cosmogenic flux. It is interesting to notice that the source-neutrinos produced in $p-\gamma$ interactions are almost comparable to the expected cosmogenic fluxes in the presented cases, while the source-neutrinos produced in $p-p$ interactions reach the level of the measured neutrino flux. This result confirms that taking into account the in-source interactions in a multi-messenger perspective might improve the constraining capability of UHECR models.

We then compute the required emissivity $\varepsilon_{\mathrm{CR}}$, i.e. the luminosity density needed to power the UHECRs at redshift $z=0$. We define $\alpha$ as the ratio between the CR luminosity $\left(L_{\mathrm{CR}}\right)$ and IR luminosity obtained from the best fit $\left(L_{\mathrm{IR}}=3.4 \cdot 10^{45} \mathrm{erg} / \mathrm{s}\right)$. In general, the IR photon field is the strongest field permeating the starburst environment. Interpreting $\alpha$ as efficiency of CR production, we assume a sub-equipartition between CR and the IR field, namely $\alpha \lesssim 10 \%$. The number density of sources, $n_{\mathrm{SBG}}$, can be estimated as

$$
n_{\mathrm{SBG}}=\frac{\varepsilon_{\mathrm{CR}}}{\alpha \cdot L_{\mathrm{IR}}}=1.8 \cdot 10^{-6}\left[\frac{\alpha}{0.1}\right]^{-1} \mathrm{Mpc}^{-3}
$$

In [23] it is shown how to model the luminosity density at a fixed redshift, using the luminosity functions $\Phi=\frac{d N}{d \log L d V_{c}}$; by integrating these functions for luminosities above the luminosity that best describes the data and considering redshift shells with $z<1$, we find:

$$
n_{\mathrm{SBG}} \simeq 3 \cdot 10^{-5} \mathrm{Mpc}^{-3}
$$

It can be observed that the number density of sources inferred from the integral of the luminosity function is an order of magnitude higher than the one obtained with the fiducial value $\alpha=10 \%$. This result is encouraging since it can be reconciled with a lower efficiency and milder dynamical impact of UHECRs in the starburst environment.

\section{Summary and future perspectives}

In this work a study of the UHECRs interactions in the environment surrounding the sources has been performed ("source-propagation model"), applied in particular to Starburst Galaxies. To this aim, we use a prototype source; therefore, once defined the interaction and escape times, we show how it is possible to find a description of the energy spectrum and mass composition measured by the Pierre Auger Observatory, connected to the features of the sources. In addition, the associated neutrino flux is computed, and we show how this improves the constraining capability of our model.

Several assumptions have been chosen in this work, such as the ones related to the distribution of the sources, that are considered identical. In addition, some simplifications are made in the computation of the interactions. Future refinements of these assumptions will render this sourcepropagation model more predictive, in terms of determination of source parameters. 
Possible future perspectives of this work also concern the possibility to produce and propagate photons inside the sources. As the neutrino fluxes, the expected photon fluxes can be compared to experimental data, possibly improving the constraining capability of our model.

\section{Acknowledgements}

We thank our colleagues from the Pierre Auger Collaboration for fruitful discussions.

\section{References}

[1] R. Aloisio, V. Berezinsky and P. Blasi, JCAP 10 (2014), 020

[2] E. Guido for the Pierre Auger Collaboration, this Conference

[3] M. Unger, G.R. Farrar and L.A. Anchordoqui, Phys. Rev. D 92, 123001 (2015)

[4] N. Globus, D. Allard and E. Parizot, Phys. Rev. D 92 (2015) no.2, 021302

[5] D. Allard and R. J. Protheroe, Astron. Astrophys. 502 (2009), 803

[6] A. D. Supanitsky, A. Cobos and A. Etchegoyen, Phys. Rev. D 98 (2018) no.10, 103016

[7] A. Aab et al. [Pierre Auger], [arXiv:1909.09073 [astro-ph.HE]].

[8] Y. Gao and P. M. Solomon, Astrophys. J. 606 (2004), 271-290,

[9] G. H. Rieke et al. Astrophys. J. 692 (2009), 556-573,

[10] R. C. Kennicutt, Jr., Astrophys. J. 498 (1998), 541

[11] E. Peretti et al. [arXiv:2104.10978 [astro-ph.HE]]

[12] G. E. Romero, A. L. Müller and M. Roth, Astron. Astrophys. 616 (2018), A57

[13] L. A. Anchordoqui, Phys. Rev. D 97 (2018) no.6, 063010

[14] A. Aab et al. [Pierre Auger], Astrophys. J. Lett. 853 (2018) no.2, L29

[15] E. Peretti et al. Mon. Not. Roy. Astron. Soc. 487 (2019) no.1, 168-180

[16] R. Aloisio et al. JCAP11(2017)009.

[17] G. R. Blumenthal, Phys. Rev. D 1 (1970), 1596-1602

[18] F. Riehn et al. Phys. Rev. D 102 (2020) no.6, 063002

[19] P. Subedi et al. Astrophys. J. 837 (2017) no.2, 140

[20] M. G. Aartsen et al. [IceCube], [arXiv:1710.01191 [astro-ph.HE]].

[21] J. Álvarez-Muñiz et al. [GRAND], Sci. China Phys. Mech. Astron. 63 (2020) no.1, 219501

[22] A. Aab et al. [Pierre Auger], JCAP 10 (2019), 022

[23] C. Gruppioni et al. Mon. Not. Roy. Astron. Soc. 432 (2013), 23 